\title{
Status of online diet management program users in Japan during the 2020 Coronavirus disease 2019 pandemic
}

\author{
Masahide Hamaguchi, ${ }^{1,+}$ Tetsuya Nojiri, ${ }^{2,+}$ Takuro Okamura, ${ }^{1}$ Yoshitaka Hashimoto, ${ }^{1}$ Akiyoshi Hanai, ${ }^{2}$ \\ Shota Narisawa, ${ }^{2}$ Emi Ushigome, ${ }^{1}$ Naoko Nakanishi, ${ }^{1}$ and Michiaki Fukui ${ }^{1, *}$ \\ 'Department of Endocrinology and Metabolism, Graduate School of Medical Science, Kyoto Prefectural University of Medicine, \\ 465 Kajii-cho, Kawaramachi-Hirokoji, Kamigyo-ku, Kyoto 602-8566, Japan \\ 2Oishi Kenko Incorporated, \&WORK NINGYOCHO, 6-4 Nihonbashi Tomizawa-cho, Chuo-ku, Tokyo 103-0006, Japan
}

(Received 3 March, 2021; Accepted 30 March, 2021; Published online 4 June, 2021)

\begin{abstract}
The spread of coronavirus disease 2019 (COVID-19) has led to drastic changes in people's lifestyles, including teleworking and restrictions on socializing. In the context of observing social distancing for preventing infection, the need to maintain fitness and health has attracted particular attention. We aimed to determine the relationship between the increase in the number of active users of online diet management applications and COVID-19 infection rates in Japan. A total of 1.5 million rows of log data was analyzed. The active number of users of online diet management applications increased with increase in the number of COVID-19 infections. The active user number in Kanagawa Prefecture, where the first cases of COVID-19 were reported in Japan, was particularly high. Moreover, there was a significant increase in the user number in prefectures under a state of emergency when compared to that in prefectures not under a state of emergency. The social anxiety caused by COVID-19 is expected to increase the demand for online health management applications further. The use of such programs can aid in achieving social distancing while enabling users to maintain healthy lifestyles.
\end{abstract}

Key Words: COVID-19, health informatics, telemedicine

$\mathrm{C}$ oronavirus disease 2019 (COVID-19), an infectious disease caused by the novel coronavirus (2019-nCoV, SARSCoV-2), was first identified in 2019 near Wuhan City, Hubei Province, China. ${ }^{(1)}$ SARS-CoV-2 has caused a global pandemic of COVID-19 owing to its highly latent viral characteristics. ${ }^{(1)}$ With the COVID-19 global pandemic, the world has entered what is now referred to as the COVID-19 era. ${ }^{(2)}$ The spread of COVID-19 has led to major changes in people's lifestyles, including teleworking and restrictions on socializing. The global economy has adapted to make the best use of information technology (IT) to ensure social distancing.

In Japan, the first state of emergency was declared on April 7, 2020, covering seven prefectures including Tokyo, Kanagawa, Osaka, and Fukuoka. On April 16, 2020, the state of emergency was extended to cover the entire country. The original emergency deadline was set for May 6; however, it was subsequently extended to May 31 for the entire country. On May 14, the state of emergency declarations for all but eight prefectures, including Tokyo, Kanagawa, and Osaka, were lifted. Subsequently, on May 25 , the state of emergency declarations for all prefectures were lifted. However, the subsequent public health situation has continued to significantly impact people's lives.

It is generally accepted that diet, exercise, and sleep are important for a healthy life. However, in the COVID-19 era, activities related to these components are changing significantly. In Japan, a stay-at-home policy has been proposed to prevent infection, and consequently, online diet management services have received considerable attention as a means of addressing the aforementioned needs. However, the association between the use of online services for diet management and the spread of COVID-19 era still needs to be studied. In Japan, several online meal management services provide advice on selecting cooking ingredients, cooking methods, and ways to "plate" food. In this context, Oishi Kenko Inc. has been offering personalized meal management services based on the user health status since 2016 . In this study, we considered the user data from the company to investigate the association between the increase in the active user number and the infection status in active user resident areas and their distance from areas under the state of emergency. The results showed an increase in the widespread use of online IT-based services for health management and monitoring driven by social anxiety and the imposition of social distancing measures during the COVID-19 pandemic.

\section{Materials and Methods}

Design. Observational study.

Setting. The logs of an online diet management application were analyzed.

Participants. Oishi Kenko Inc. (https://oishi-kenko.com/) offers a dietitian-directed recipe search and menu creation service for the prevention and management of diseases as well as weight loss via web and mobile applications (iOS and Android). In this study, we analyzed the logs of an online diet management application. This study was approved by Kyoto Prefectural University of Medicine, Clinical Research Review Board (ERB-C-1638). The consent for this study was obtained by the opt-out approach. Oishi Kenko Inc. declares that data obtained from the service is to be used for research related to healthcare as per Article 10 "Utilization of usage status, etc." on its website (https://oishikenko.com/terms). If the user does not agree with the declaration during registration for the use of the service, they can choose not to register. Existing users who disagree with these terms can express their refusal in the form of withdrawal from the service.

In this study, we used data from both the web and mobile

${ }^{\dagger}$ These authors contributed equally to this article.

* To whom correspondence should be addressed.

E-mail: michiaki@koto.kpu-m.ac.jp 
applications. We re-moved individual-identifiable information from the data, and the anonymized active user information, menu preparation information, and recipe search information were exported. The number of active users was calculated for each ISO week from W01 (Week 1) to W21 (Week 21), 2020.

COVID-19 infection information was obtained from reports on the number of COVID-19 cases (outbreaks, domestic outbreaks, airport quarantine cases, overseas situation, and total number of COVID-19 cases) in Japan as provided by the Ministry of Health, Labour and Welfare (MHLW) from Toyo Keizai under the MIT license. (3,4) $^{(3,}$

The population and number of households in each prefecture and the prefecture location were used to characterize the area of residence of the active users. The estimate of the population in a prefecture was based on population estimates as of October 1, $2017 .{ }^{(5)}$ The number of households by prefecture was based on the 2019 estimates of the number of households in Japan. ${ }^{(6)}$ Distances between prefectures were estimated based on straightline point-to-point distances between prefectural offices. ${ }^{(7)}$

Definitions. In this study, we analyzed the data corresponding to the duration from ISO W01 to W21, 2020. The first COVID-19 infection in Japan was identified on February 1 (ISO W05), 2020, in Kanagawa Prefecture. Weeks W01-W04 were defined as the pre-COVID-19 era, and we used the data of W03 as a reference for the pre-COVID-19 era in Japan. Moreover, we defined the COVID-19 era as W05-W21 and the duration of the state of emergency as W15-W21. In Japan, the first state of emergency was declared on April 7 (ISO W15), covering seven prefectures including Tokyo, Kanagawa, Osaka, and Fukuoka. On May 25 (first day of ISO W22), the state of emergency declarations for all prefectures were lifted.

The cumulative number of infected people during the state of emergency was estimated on April 12, 2020 (last day of ISO $\mathrm{W} 15)$. The number of newly infected people during the state of emergency was defined as the number of newly infected people in April 5-12, 2020 (ISO W15). The cumulative infection rate during the state of emergency was defined as the cumulative number of infected people as estimated on April 12, 2020, divided by the population of the province. The rate of new infections during the state of emergency was calculated as the number of new infections divided by the population of the prefecture in April 5-12, 2020 (ISO W15).

The active user number was standardized by the number of active users per 1,000 households in the prefecture. The number of active online diet management application users before the COVID-19 era was defined as the number of active users in the week of January 13-19, 2020 (ISO W03). The active user number during the state of emergency was defined as that in the week of April 5-12, 2020 (ISO W15). We also estimated the rate of new infections and the active user number in the week of May 18-24, 2020 (ISO W21). The increase in the active user number during the state of emergency was defined as the number of active users per 1,000 households at ISO W15 or ISO W21 subtracted by the number of active users per 1,000 households at ISO W03.

Statistical methods. We conducted a $t$ test for continuous variables between the two types of areas considered (areas covered by the state of emergency and those not covered by the emergency), and we conducted a chi-squared test to evaluate the differences in pro-portions between the covered and non-covered areas. To clarify the association between the number of active users per 1,000 households and the cumulative infection rate or geographical distance from Tokyo (Japan's capital city, which exhibited the highest cumulative infection rate) or the prefectures wherein a state of emergency was declared for COVID-19, we generated scatterplots and asymptotic lines. The associations were evaluated by means of Pearson's correlation coefficient. A $p$ value of $\leq 0.05$ was considered statistically significant. We analyzed all data using SPSS software (ver. 25; SPSS Inc., Chicago, IL). In the study, the continuous variables are presented as means with SD.

\section{Results}

We analyzed a total of 1.5 million rows of $\log$ data and conducted a spatial epidemiological study of the number of active users per 1,000 households. Changes in the cumulative infection rate per population and the number of active users per 1,000 households in each prefecture were projected onto a map of Japan (Fig. 1). During ISO W03, no COVID-19 infections were identified in Japan except in Kanagawa Prefecture (Fig. 1 and Supplemental Fig. 1*). After ISO W04, the number of COVID-19 patients in Japan increased gradually, and the infection began to spread to different prefectures (Supplemental Fig. 1*). Simultaneously, the number of active online diet program users per household increased (Fig. 1). Figure 2 displays the trends in infections and active users over time. It can be observed that the number of active users per 1,000 households increased along with the cumulative infection rate per population. After ISO W13, the rate of infection increased dramatically in some prefectures, with Tokyo exhibiting the highest cumulative infection rate (Fig. 2). Subsequently, the number of active users per 1,000 households increased in some prefectures (Fig. 2).

The number of active users per 1,000 households increased significantly in the prefectures under a state of emergency, as listed in Table 1. The number of active users doubled after the declaration of the state of emergency from 80,011 during ISO W03 to 104,282 during ISO W15, corresponding to an increase of over 20,000 people. There were 1.96 active users per 1,000 households during ISO W15 across Japan. The prefectures under a state of emergency had a higher total number of households and a larger population; therefore, these prefectures had significantly higher cumulative infection rates. The number of active users per 1,000 households also increased significantly in prefectures under the state of emergency when compared with those not under emergency.

Next, we examined the relationship between the cumulative infection rate per population and the increase in the number of active users per 1,000 households (Fig. 3). The number of active users per 1,000 households during the state of emergency or during the COVID-19 era increased with the cumulative infection rate. An increase was observed in the number of active users per 1,000 households during the state of emergency or during the COVID-19 era in both prefectures under and not under the state of emergency as the cumulative infection rate increased. In particular, the number of active users per 1,000 households increased prominently in Kanagawa Prefecture; however, this increase was observed to occur earlier than in the other cases (Fig. 2).

We also found that the number of active users per 1,000 households during the state of emergency or during the COVID-19 era was inversely correlated with the geographical distance from Tokyo, which had the highest cumulative infection rate. Moreover, the number of active users per 1,000 households was correlated with the geographical distance from Tokyo (Supplemental Fig. $2 *$ )

Next, we evaluated the increase in the number of active users per 1,000 households in relation to their geographical distance to the seven prefectures under the state of emergency (Supplemental Fig. 3*). The results showed that the geographical distance from the seven prefectures increased with increase in the number of active users per 1,000 households. The geographical distances between the prefectures near Tokyo and the seven prefectures under emergency were correlated with the cumulative number of people infected per population (Supplemental Fig. 2 and 3*). 
W15
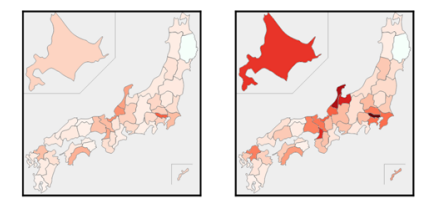

15

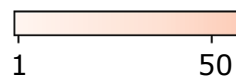

50
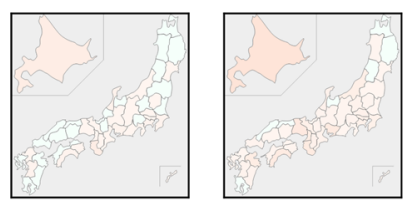

150

200
250

300

the umber per 1 million

B

The geological changes in the number of active users for "online diet management application" per households in Japan

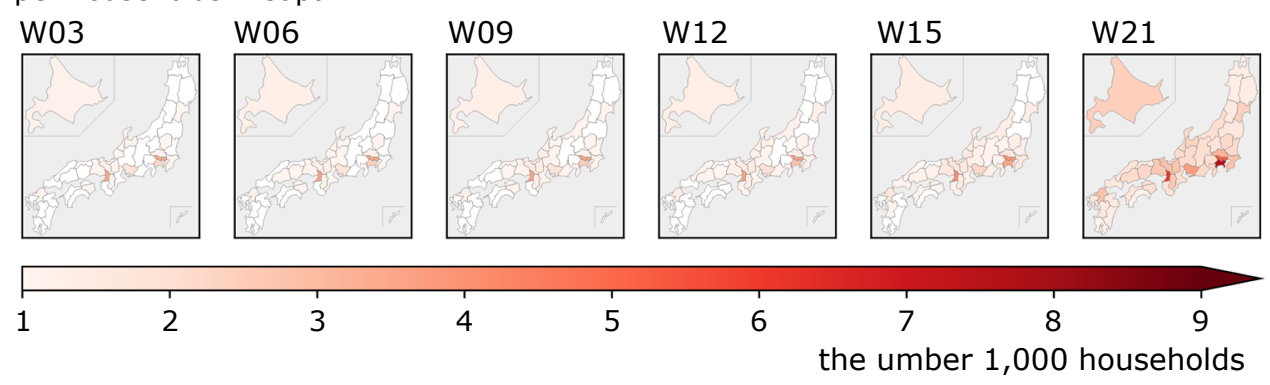

Fig. 1. The geospatial changes in the cumulative COVID-19 infection rate per population and in the number of online diet management application active users in Japan. Right panel shows the reference map. Prefectures under a state of emergency declaration are colored orange; Kanagawa Prefecture is colored pink; and prefectures not under a state of emergency declaration are colored blue. (A) The geospatial changes in the cumulative infection rate of COVID-19 per population in Japan shown in different shades of red. The cumulative infection rate is expressed per a million people. (B) The geospatial changes in the number of active users for online diet management application per households in Japan shown in different shades of red. The number of active users is expressed per 1,000 households. See color figure in the on-line version.

\section{Discussion}

The number of active users of online diet management applications increased during the COVID-19 era. There was a significant increase in the active user number in areas under a state of emergency, and this number increased according to the cumulative infection rate in the region. These results suggest that the active user number will continue to increase owing to the social anxiety associated with COVID-19 infection rates in the region and the stay-at-home policy implemented as a consequence of the declaration of a state of emergency.

In addition, the number of active users per household increased significantly, particularly in Kanagawa Prefecture. Although the reason for this significant increase in Kanagawa Prefecture was unclear, we hypothesize that this increase was influenced by social anxiety regarding the relatively higher number of COVID-19 infections in this region. The first COVID-19 infection in Japan was identified on February 1, 2020, in Kanagawa Prefecture. This octogenarian patient was onboard the cruise ship Diamond Princess, which departed from the Port of Yokohama on January 20, 2020. The patient subsequently disembarked in Hong Kong on January 25, 2020.(8) SARS-CoV-2 RNA was detected in 10 of 31 Diamond Princess crew members on February 4, 2020. ${ }^{(8)}$ A total of 712 out of 3,713 passengers tested positive for COVID-19 by April 15, 2020, and at least 13 deaths were confirmed in Kanagawa Prefecture (case fatality rate of $1.8 \%$ ). ${ }^{(9)}$ The fact that deaths were actually occurring in Kanagawa Prefecture owing to COVID-19 suggests that social anxiety in the prefecture was more pronounced than in other areas. Thus, Kanagawa Prefecture differed from the other prefectures in that it had confirmed positive SARS-CoV-2 RNA infections as of February 4, 2020.

There have been various reports on the characteristics of host that aggravate the severity of COVID-19 symptoms. ${ }^{(10-26)}$ Aging over 65 years, malignant tumor, chronic obstructive pulmonary disease, chronic kidney disease, type 2 diabetes, hypertension, dyslipidemia, obesity, smoking, or immune deficiency is thought to be associated with the severity of COVID-19 symptoms. ${ }^{(10-26)}$ In regard with a condition for immune deficiency, selective IgA deficiency can be associated with COVID-19 the severity of COVID-19 symptoms. ${ }^{(27)}$ In addition to underlying disease of the host, nutritional states of host are also associated with the severity of COVID-19 symptoms. ${ }^{(28,29)}$ Malnutrition and hypoproteinemia were reported to be associated with the severity of COVID-19 symptoms. ${ }^{(28,29)}$ Not only is malnutrition a risk for the severe, but overnutrition is a risk for the severity as well. ${ }^{(10,11)} \mathrm{In}$ particular people with diabetes and obesity are reportedly at greater risk for severe COVID-19 symptoms. ${ }^{(17,30-34)}$ Therefore, in the COVID-19 era, the prevention and treatment of diabetes and other lifestyle diseases assumes even greater importance. Conversely, during the COVID-19 era, the stay-at-home policy has led to significant changes in people's life-styles. Therefore, lifestyle interventions optimized for this new reality involving COVID-19 are inevitable.

In fact, changes in exercise and eating habits were reported in diabetic patients owing to anxiety about COVID-19, with diabetics experiencing a worsening of $\mathrm{HbA1C}$ levels and increasing body weight. ${ }^{(35)}$ Thus, effective dietary management is required for people with comorbidities during the COVID-19 era. In this context, the current study indicates that the COVID-19 infection rate in a given region, the declaration of a state of emergency, and the stay-at-home policy led to an increase in the 
A

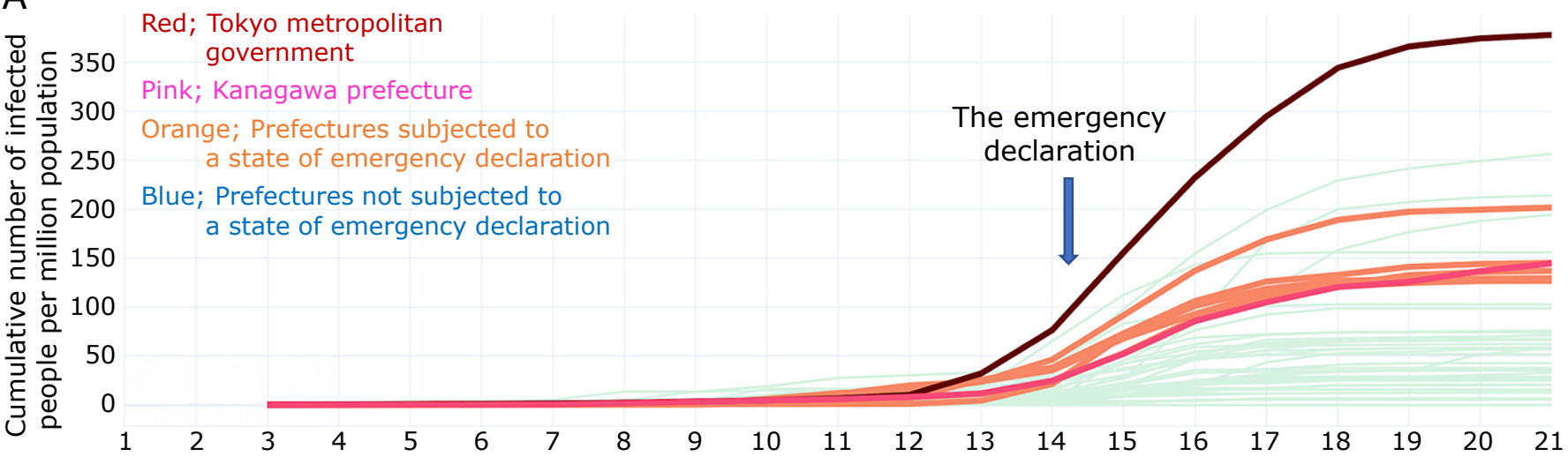
ISO week

Region - Aichi - Akita Aomori Ehime Fukui Fukushima Gifu - Gunma Hiroshima Hokkaido - Ibaraki- Ishikawa Iwate

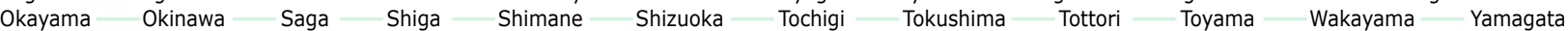
Yamaguchi Yamanashi $\rightleftharpoons$ Chiba $\rightleftharpoons$ Fukuoka Hyogo $\rightleftharpoons$ Osaka $\rightleftharpoons$ Saitama $\rightleftharpoons$ Tokyo Kanawa

B

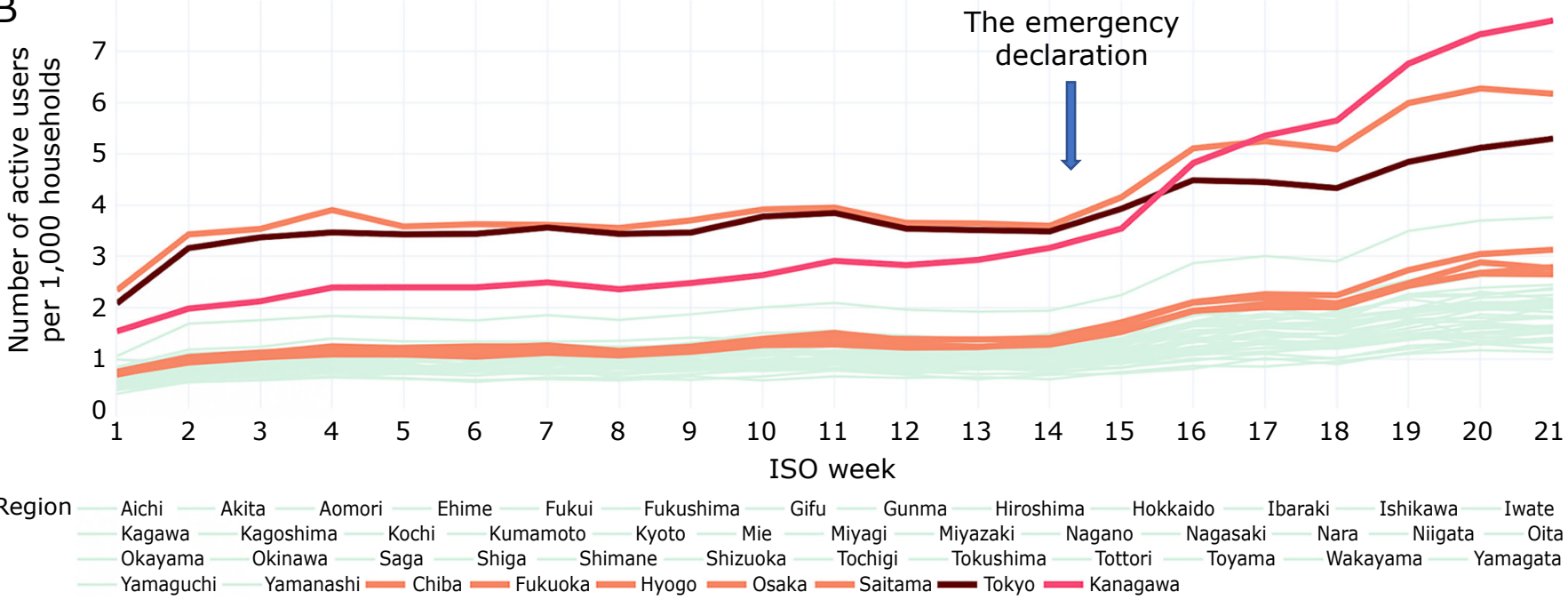

Fig. 2. The transient of number of active users per 1,000 households and the number of cumulative COVID-19 infections per total population in each prefecture. (A) The cumulative infection rate expressed per 1 million people. (B) The number of active users expressed per 1,000 households. Orange color represents prefectures under a state of emergency declaration; pink color represents Kanagawa Prefecture; and blue color represents prefectures under a state of emergency declaration. See color figure in the on-line version.

Table 1. Basic characteristics between prefectures under a state of emergency and those with no state of emergency

\begin{tabular}{|c|c|c|c|}
\hline & $\begin{array}{l}\text { Prefecture not subject } \\
\text { to a state of } \\
\text { emergency declaration }\end{array}$ & $\begin{array}{c}\text { Prefecture subject } \\
\text { to a state of } \\
\text { emergency declaration }\end{array}$ & $p$ value \\
\hline Number of prefectures & 40 & 7 & \\
\hline Total number of households in the prefecture $(\times 1,000)$ & $716.9(552.3)$ & $3,522.3(1,568.6)$ & 0.003 \\
\hline Cumulative Infections of COVID-19 in the prefecture & $54.4(67.5)$ & $713.1(641.2)$ & 0.035 \\
\hline Cumulative Infections of COVID-19 per number of people & $29.2(25.7)$ & $80.7(35.8)$ & $<0.001$ \\
\hline Number of active users before COVID-19 era & $724.1(887.2)$ & $7,968.9(7,717.1)$ & 0.048 \\
\hline Number of active users per 1,000 households after COVID-19 era & $1.2(0.3)$ & $2.6(1.2)$ & 0.021 \\
\hline Number of active users per 1,000 households before COVID-19 era & $0.9(0.2)$ & $1.9(1.1)$ & 0.056 \\
\hline $\begin{array}{l}\text { Increased number of active users per } 1,000 \text { households before and } \\
\text { after COVID-19 era }\end{array}$ & $0.3(0.1)$ & $0.7(0.3)$ & 0.014 \\
\hline
\end{tabular}

Data expressed as means (SD). A t test was applied to assess the difference between two groups. 
A

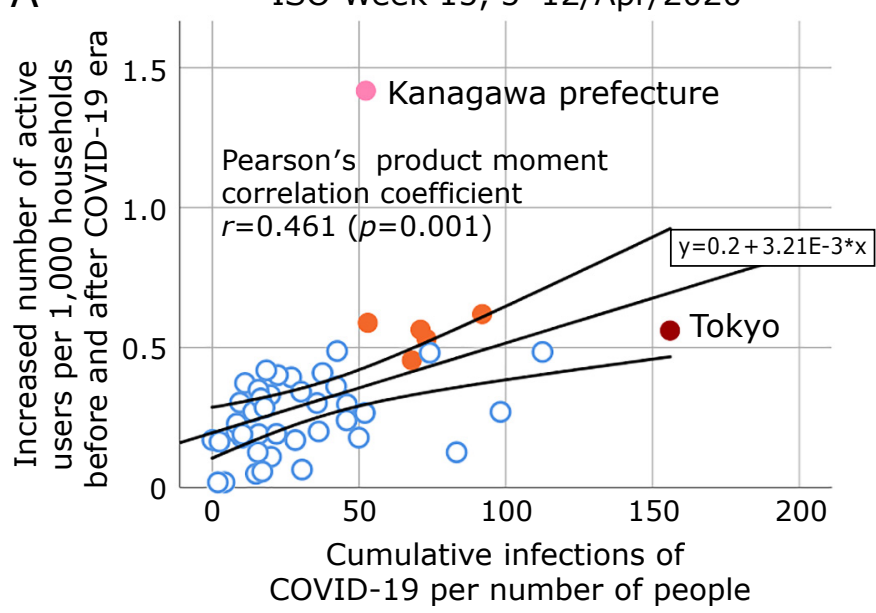

B

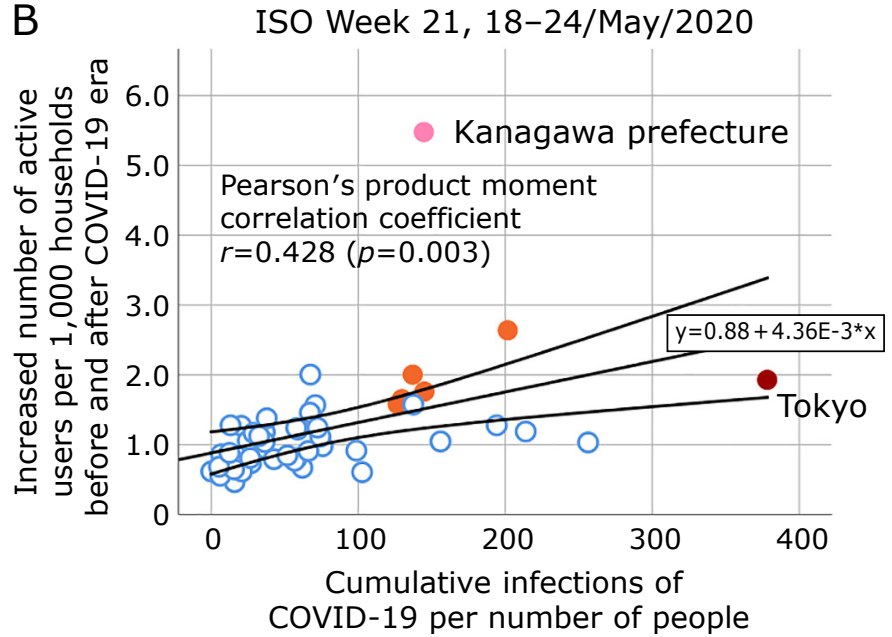

Fig. 3. The association between cumulative COVID-19 infections per number of people and the increase in the number of active users in prefectures under a state of emergency during the COVID-19 era. (A) The increase in the number of active users was calculated as the number of users in ISO W15 minus the number of users in ISO W03. (B) The increase in the number of active users was calculated as the number of users in ISO W21 minus the number of users in ISO W03. The cumulative infection rate is expressed per 1 million people. The number of active users is expressed per 1,000 households.

number of users of online dietary management applications. Therefore, diet management through online meal management apps has become a new approach for addressing people's health needs in the COVID-19 era.

This is the first study to examine the association between the COVID-19 pan-demic and the use of online dietary management applications from a spatial epidemiological perspective. However, the study suffers from certain limitations: The applicability of these results to other countries may be limited as the data as well as the app are limited to Japan. Another limitation of this study is that psychological factors were not considered. Although social anxiety caused by the COVID-19 pandemic is speculated to have contributed to the increase in the number of active users of online dietary management applications, no psychological assessment of the population was conducted. Authors should discuss the results and how they can be interpreted from the perspective of previous studies and of the working hypotheses. The findings and their implications should be discussed in the broadest context possible. Future research directions may also be highlighted.

\section{Conclusions}

In our study, we determined that there has been a significant increase in the number of users of online dietary management applications owing to social anxiety and the stay-at-home policies implemented in the COVID-19 era. We note here that the optimization of lifestyle interventions has become essential for the health and well-being of people in the COVID-19 era. Online healthcare services are expected to be further expanded, and therefore, their usefulness needs to be thoroughly tested.

\section{Author Contributions}

Conceptualization, $\mathrm{MH}$ and $\mathrm{MF}$; methodology, $\mathrm{MH}, \mathrm{TN}, \mathrm{AH}$, and $\mathrm{SN}$; software, $\mathrm{TN}, \mathrm{AH}$, and $\mathrm{SN}$; validation, $\mathrm{EU}$ and $\mathrm{NN}$; formal analysis, $\mathrm{MH}$; investigation, $\mathrm{MH}$; resources, $\mathrm{TN}$; data curation, $\mathrm{TN}, \mathrm{AH}$, and $\mathrm{SN}$; writing-original draft preparation, $\mathrm{MH}$; writing-review and editing, $\mathrm{TO}$ and $\mathrm{YH}$; visualization, $\mathrm{AH}$; supervision, $\mathrm{MF}$; project administration, $\mathrm{MF}$; funding acquisition, MF. All authors have read and agreed to the published version of the manuscript.

\section{Acknowledgments}

This research received funding from Oishi Kenko Inc. The funder had no role in the design and conduct of the study; collection, management, analysis and interpretation of data; preparation, review, or approval of the manuscript; and decision to submit the manuscript for publication. We thank all of the staff members of the Kyoto Prefectural University of Medicine. We would like to thank Editage (www.editage.com) for English language editing.

\section{Conflict of Interest}

All authors have completed the ICMJE uniform disclosure form at www.icmje.org/coi_disclosure.pdf and declare the following: $\mathrm{MH}$ has received grants from Asahi Kasei Pharma, Nippon Boehringer Ingelheim Co., Ltd., Mitsubishi Tanabe Pharma Corporation, Daiichi Sankyo Co., Ltd., Sanofi K.K., Takeda Pharmaceutical Company Limited, Astellas Pharma Inc., Kyowa Kirin Co., Ltd., Sumitomo Dainippon Pharma Co., Ltd., Novo Nordisk Pharma Ltd., and Eli Lilly Japan K.K., outside the submitted work.

YH has received grants from Asahi Kasei Pharma and personal fees from Daiichi Sankyo Co., Ltd., Mitsubishi Tanabe Pharma Corp., Sanofi K.K., and Novo Nordisk Pharma Ltd., outside the submitted work.

EU has received grants from the Japanese Study Group for Physiology and Management of Blood Pressure and the Astellas Foundation for Research on Metabolic Disorders (Grant number: 4024). The Donated Fund Laboratory of Diabetes therapeutics is an endowment department, supported with an unrestricted grant from Ono Pharmaceutical Co., Ltd.,

EU received personal fees from AstraZeneca PLC, Astellas Pharma Inc., Daiichi Sankyo Co., Ltd., Kyowa Hakko Kirin Company Ltd., Kowa Pharmaceutical Co., Ltd., MSD K.K., Mitsubishi Tanabe Pharma Corp., Novo Nordisk Pharma Ltd., Taisho Toyama Pharmaceutical Co., Ltd., Takeda Pharmaceutical Co., Ltd., Nippon Boehringer Ingelheim Co., Ltd., and Sumitomo Dainippon Pharma Co., Ltd., outside the submitted work.

MF has received grants from Nippon Boehringer Ingelheim Co., Ltd., Kissei Pharma Co., Ltd., Mitsubishi Tanabe Pharma Corp., Daiichi Sankyo Co., Ltd., Sanofi K.K., Takeda Pharma 
Co., Ltd., Astellas Pharma Inc., MSD K.K., Kyowa Hakko Kirin Co., Ltd., Sumitomo Dainippon Pharma Co., Ltd., Kowa Pharmaceutical Co., Ltd., Novo Nordisk Pharma Ltd., Ono Pharma Co., Ltd., Sanwa Kagaku Kenkyusho Co., Ltd., Eli Lilly Japan K.K., Taisho Pharma Co., Ltd., Terumo Co., Teijin Pharma Ltd., Nippon Chemiphar Co., Ltd., and Johnson \& Johnson K.K. Medical Co., Abbott Japan Co., Ltd.

MF received personal fees from Nippon Boehringer Ingelheim Co., Ltd., Kissei Pharma Co., Ltd., Mitsubishi Tanabe Pharma Corp., Daiichi Sankyo Co., Ltd., Sanofi K.K., Takeda Pharma Co., Ltd., Astellas Pharma Inc., MSD K.K., Kyowa Kirin Co., Ltd., Sumitomo Dainippon Pharma Co., Ltd., Kowa Pharma Co.,

\section{References}

1 Zhou P, Yang XL, Wang XG, et al. A pneumonia outbreak associated with a new coronavirus of probable bat origin. Nature 2020; 579: 270-273.

2 Gern KJ, Hauber P, Stolzenburg U. Business cycle highlight: global corona shock on the labour market. Wirtschaftsdienst 2020; 100: 387-388. (in German)

3 Ministry of Health, Labour and Welfare. https://www.mhlw.go.jp/stf/ seisakunitsuite/bunya/0000121431_00086.html. Accessed 14 Jul 2020.

4 Ogiwara K. https://github.com/kaz-ogiwara/covid19/blob/master/data/ prefectures.csv. Accessed 14 Jul 2020.

5 Official Statistics of Japan. https://www.e-stat.go.jp/dbview?sid=0003215867. Accessed 14 Jul 2020.

6 National Institute of Population and Social Security Research. http:// www.ipss.go.jp/pp-pjsetai/j/hpjp2019/gaiyo/data.asp. Accessed 14 Jul 2020.

7 Geospatial Information Authority of Japan. https://www.gsi.go.jp/ KOKUJYOHO/kenchokan.html. Accessed 14 Jul 2020.

8 Kakimoto K, Kamiya H, Yamagishi T, Matsui T, Suzuki M, Wakita T. Initial investigation of transmission of COVID-19 among crew members during quarantine of a cruise ship_-Yokohama, Japan, February 2020. MMWR Morb Mortal Wkly Rep 2020; 69: 312-313.

9 Tokuda Y, Sakihama T, Aoki M, et al. COVID-19 outbreak on the Diamond Princess Cruise Ship in February 2020. J Gen Fam Med 2020; 21: 95-97.

10 Richardson S, Hirsch JS, Narasimhan M, et al. Presenting characteristics, comorbidities, and outcomes among 5700 patients hospitalized with COVID-19 in the New York City area. JAMA 2020; 323: 2052-2059.

11 Cummings MJ, Baldwin MR, Abrams D, et al. Epidemiology, clinical course, and outcomes of critically ill adults with COVID-19 in New York City: a prospective cohort study. Lancet 2020; 395: 1763-1770.

12 Matsunaga N, Hayakawa K, Terada M, et al. Clinical epidemiology of hospitalized patients with COVID-19 in Japan: report of the COVID-19 registry Japan. Clin Infect Dis 2020; ciaa1470.

13 Saito S, Asai Y, Matsunaga N, et al. First and second COVID-19 waves in Japan: a comparison of disease severity and characteristics. J Infect 2021; 82: 84-123.

14 Liang W, Guan W, Chen R, et al. Cancer patients in SARS-CoV-2 infection: a nationwide analysis in China. Lancet Oncol 2020; 21: 335-337.

15 Lippi G, Henry BM. Chronic obstructive pulmonary disease is associated with severe coronavirus disease 2019 (COVID-19). Respir Med 2020; 167: 105941

16 Myers LC, Parodi SM, Escobar GJ, Liu VX. Characteristics of hospitalized adults with COVID-19 in an integrated health care system in California. JAMA 2020; 323: 2195-2198.

17 Fadini GP, Morieri ML, Longato E, Avogaro A. Prevalence and impact of diabetes among people infected with SARS-CoV-2. J Endocrinol Invest 2020; 43: 867-869.

18 Zheng Z, Peng F, Xu B, et al. Risk factors of critical \& mortal COVID-19 cases: a systematic literature review and meta-analysis. J Infect 2020; 81: e16-e25.

19 Yang J, Zheng Y, Gou X, et al. Prevalence of comorbidities and its effects in patients infected with SARS-CoV-2: a systematic review and meta-analysis. Int J Infect Dis 2020; 94: 91-95.

20 Popkin BM, Du S, Green WD, et al. Individuals with obesity and COVID-19: a global perspective on the epidemiology and biological relationships. Obes Rev 2020; 21: e13128
Ltd., Novo Nordisk Pharma Ltd., Ono Pharma Co., Ltd., Sanwa Kagaku Kenkyusho Co., Ltd., Eli Lilly Japan K.K., Taisho Pharma Co., Ltd., Bayer Yakuhin, Ltd., AstraZeneca K.K., Mochida Pharma Co., Ltd., Abbott Japan Co., Ltd., Medtronic Japan Co., Ltd., Arkley Inc., Teijin Pharma Ltd., and Nipro Co., outside the submitted work.

\section{Data Availability Statement}

The original contributions presented in the study are included in the article/Supplementary Material, further inquiries can be directed to the corresponding author.
21 Latif F, Farr MA, Clerkin KJ, et al. Characteristics and outcomes of recipients of heart transplant with coronavirus disease 2019. JAMA Cardiol 2020; e202159.

22 Brenner EJ, Ungaro RC, Gearry RB, et al. Corticosteroids, but not TNF antagonists, are associated with adverse COVID-19 outcomes in patients with inflammatory bowel diseases: results from an international registry. Gastroenterology 2020; 159: 481-491

23 Michelena X, Borrell H, López-Corbeto M, et al. Incidence of COVID-19 in a cohort of adult and paediatric patients with rheumatic diseases treated with targeted biologic and synthetic disease-modifying anti-rheumatic drugs. Semin Arthritis Rheum 2020; 50: 564-570.

24 Hadi YB, Naqvi SFZ, Kupec JT, Sarwari AR. Characteristics and outcomes of COVID-19 in patients with HIV: a multicentre research network study. AIDS 2020; 34: F3-F8.

25 Ellington S, Strid P, Tong VT, et al. Characteristics of women of reproductive age with laboratory-confirmed SARS-CoV-2 infection by pregnancy status United States, January 22-June 7, 2020. MMWR Morb Mortal Wkly Rep 2020; 69: 769-775.

26 Allotey J, Stallings E, Bonet M, et al. Clinical manifestations, risk factors, and maternal and perinatal outcomes of coronavirus disease 2019 in pregnancy: living systematic review and meta-analysis. BMJ 2020; 370: $\mathrm{m} 3320$.

27 NaitoY, Takagi T, Yamamoto T, Watanabe S. Association between selective IgA deficiency and COVID-19. J Clin Biochem Nutr 2020; 67: 122-125.

28 Wei C, Liu Y, Li Y, Zhang Y, Zhong M, Meng X. Evaluation of the nutritional status in patients with COVID-19. J Clin Biochem Nutr 2020; 67: 116 121.

29 Lin L, Hu K, Cai S, et al. Hypoproteinemia is an independent risk factor for the prognosis of severe COVID-19 patients. J Clin Biochem Nutr 2020; 67: 126-130.

30 Zhu L, She ZG, Cheng X, et al. Association of blood glucose control and outcomes in patients with COVID-19 and pre-existing type 2 diabetes. Cell Metab 2020; 31: 1068-1077.e3.

31 Cariou B, Hadjadj S, Wargny M, et al. Phenotypic characteristics and prognosis of inpatients with COVID-19 and diabetes: the CORONADO study. Diabetologia 2020; 63: 1500-1515.

32 Ushigome E, Yamazaki M, Hamaguchi M, et al. Usefulness and safety of remote continuous glucose monitoring for a severe COVID-19 patient with diabetes. Diabetes Technol Ther 2021; 23: 78-80.

33 Frank RC, Mendez SR, Stevenson EK, Guseh JS, Chung M, Silverman MG. Obesity and the risk of intubation or death in patients with coronavirus disease 2019. Crit Care Med 2020; 48: e1097-e1101.

34 Al-Sabah S, Al-Haddad M, Al-Youha S, Jamal M, Almazeedi S. COVID-19: impact of obesity and diabetes on disease severity. Clin Obes 2020; 10: e12414.

35 Munekawa C, Hosomi Y, Hashimoto Y, et al. Effect of coronavirus disease 2019 pandemic on the lifestyle and glycemic control in patients with type 2 diabetes: a cross-section and retrospective cohort study. Endocr $J$ 2021; 68 : 201-210. 